PROCEEDINGS OF THE

AMERICAN MATHEMATICAL SOCIETY

Volume 125, Number 8, August 1997, Pages 2363-2371

S 0002-9939(97)03860-4

\title{
A REMARKABLE REARRANGEMENT OF THE HAAR SYSTEM IN $L_{p}$
}

\author{
PAUL F. X. MÜLLER AND GIDEON SCHECHTMAN
}

(Communicated by Dale Alspach)

\begin{abstract}
We introduce a non-standard but, to our opinion natural, order on the initial segments of the Haar system and investigate the isomorphic classification of the linear span, in $L_{p}$, of block bases, with respect to this order.
\end{abstract}

\section{INTRODUCTION}

In [DS] it was proved that every unconditional basic sequence $\left\{x_{i}\right\}_{i=1}^{\infty}$ in $L_{p}$, $2<p<\infty$, which is not equivalent to the natural basis of $\ell_{p}$ has the property that for some $K \geq 1$ and every positive integer $n$ there are $n$ vectors of the form $y_{i}=\sum_{j \in \sigma_{i}} a_{j} x_{j}, i=1, \ldots, n$, where the sets $\sigma_{i}$ are pairwise disjoint and the sequence $\left\{y_{i}\right\}$ is equivalent, with constant $K$, to the unit vector basis in $\ell_{2}^{n}$. See also [JMST] for a generalization of this fact for more general lattices. It was left open in these two papers (and specifically asked in Problem 3.A of [DS]) whether $\left\{y_{i}\right\}$ can be chosen to be a block basis, i.e., whether the sets $\sigma_{i}$ can be chosen to be successive, that is, $\max \left\{j ; j \in \sigma_{k}\right\}<\min \left\{j ; j \in \sigma_{l}\right\}$ for all $k<l$.

The initial motivation of this paper was to solve this question in the negative. As is well known the Haar basis, $\left\{h_{n, i}\right\}_{n=0, i=1}^{\infty}$, in $L_{p}(0,1), 1<p<\infty$, in its common order, has a block basis (i.e., the Rademacher functions) equivalent to the unit vector basis of $\ell_{2}$. It follows from the main results of this paper (Theorems 2.2 and 3.1) that the initial segments of the Haar system can be rearranged so that they will not have (the finite version of) this property anymore. We denote the new order by $\preceq$.

Note that the space $\left(\sum_{N=1}^{\infty} \oplus\left[h_{n, i}\right]_{n=0, i=1}^{N}\right)_{p}$ is isomorphic to $\ell_{p}$. (Here $\left[x_{i}\right]_{i \in A}$ denotes the closed linear span of $\left\{x_{i}\right\}_{i \in A}$.) Let $\left\{b_{i}\right\}_{i=1}^{\infty}$ be the natural (relative to the order $\preceq$ ) basis of $\left(\sum_{N=1}^{\infty} \oplus\left[h_{n, i}\right]_{n=0, i=1}^{N}\right)_{p}$, i.e., the basis composed of the successive conjunction of the bases $\left\{h_{n, i}\right\}_{n=0, i=1}^{N}$, each in its $\preceq$ - order. Clearly, $\left\{b_{i}\right\}_{i=1}^{\infty}$ is not equivalent to the natural basis of $\ell_{p}$ as long as $p \neq 2$, and it easily follows from the previous paragraph that

Received by the editors September 5, 1995 and, in revised form, February 23, 1996.

1991 Mathematics Subject Classification. Primary 46B07, 60G42.

The first author was supported by the Austrian Academy of Sciences (APART Program).

The second author was supported in part by BSF.

Both authors participated in the Workshop in Linear Analysis and Probability, Texas A\&M University.

(C) 1997 American Mathematical Society 
- For each $p, 1<p \neq 2<\infty$, and for each $K, 1 \leq K<\infty$, there is an $n$ such that no block basis of $\left\{b_{i}\right\}_{i=1}^{\infty}$ of length $n$ is $K$-equivalent to the unit vector basis of $\ell_{2}^{n}$.

Thus, in the case $2<p<\infty$, the sequence $\left\{b_{i}\right\}_{i=1}^{\infty}$ gives the required counterexample to the problem above.

In Section 1 below we introduce the new order on the initial segments of the Haar system and determine some of its properties. In Section 2 we prove the main theorem (2.2) which states that, for $2<p<\infty$, every block basis of such an initial segment of the Haar system is well equivalent to a diagonal of the unit vector basis of $\ell_{p}^{k}$ and a subsequence of a permutation of the Haar system. In Section 3 we deal with the case $1<p<2$ and prove a somewhat weaker property of such block bases in Theorem 3.1 but which is also enough to deduce $\bullet$ above.

\section{THE ORDER}

An interval of the form $I=\left[\frac{i-1}{2^{n}}, \frac{i}{2^{n}}\right), n=0,1, \ldots, i=1,2, \ldots, 2^{n}$, will be called a dyadic interval. The left half of the interval $I=\left[\frac{i-1}{2^{n}}, \frac{i}{2^{n}}\right)$ is the interval $\left[\frac{2 i-2}{2^{n+1}}, \frac{2 i-1}{2^{n+1}}\right)$ and the right half of $I$ is $\left[\frac{2 i-1}{2^{n+1}}, \frac{2 i}{2^{n+1}}\right)$. The Haar function $h_{I}=h_{n, i}$ associated with $I$ is the function

$$
h_{I}(t)=\left\{\begin{aligned}
1, & \text { if } t \text { is in the left half of } I, \\
-1, & \text { if } t \text { is in the right half of } I .
\end{aligned}\right.
$$

The common order on $\left\{h_{I}\right\}$ is the lexicographic order on $\{(n, i)\}$. Fixing $N$ we would like to introduce a different order, $\preceq$, on the set $T=T_{N}$ of dyadic intervals of length larger than or equal to $2^{-N}$.

Definition. $I \preceq J$ if either $I$ and $J$ are disjoint and $I$ is to the left of $J$, or, $I$ is contained in $J$.

In terms of the natural dyadic tree structure of $T$, Figure 1 describes the order, for $N=4$.

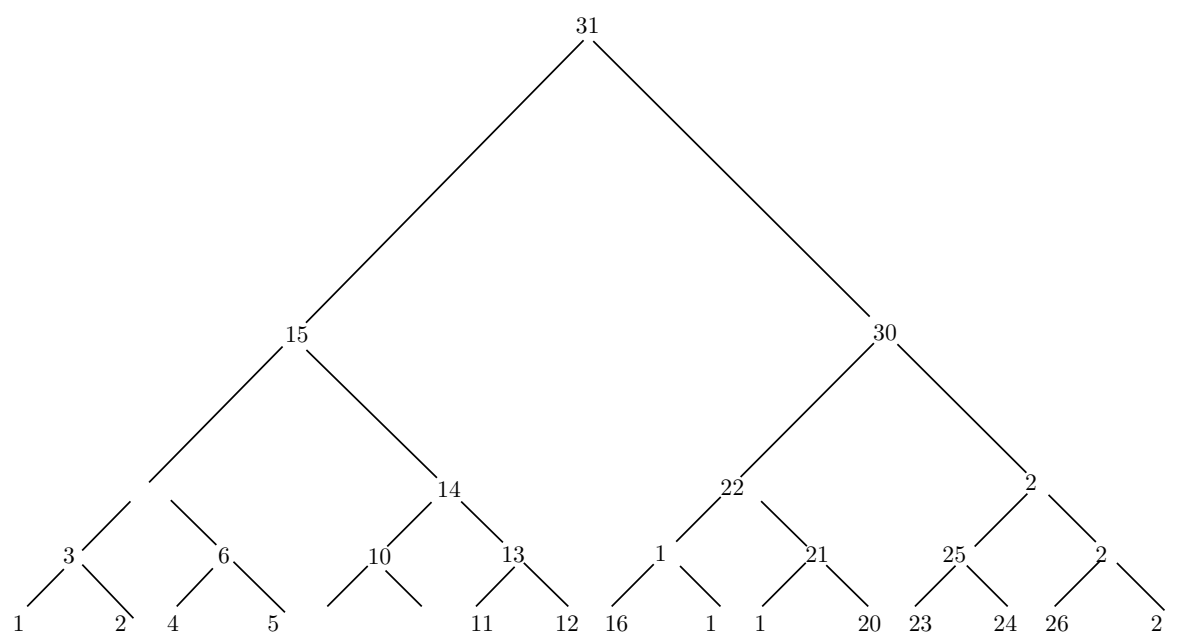

Figure 1 
Proposition 1.1 below describes the order intervals with respect to the order $\preceq$. We first need two definitions.

Definition. Given two dyadic intervals $I \subseteq J$

1. The cone $\mathcal{C}=\mathcal{C}(I, J)$ of dyadic intervals between $I$ and $J$ is the unique collection of dyadic intervals, $\mathcal{C}=\left\{C_{1}, \ldots, C_{k}\right\}$, satisfying

a) $k=\log _{2} \frac{|J|}{|I|}+1$

b) $C_{1}=I, C_{k}=J$

c) $\left|C_{s}\right|=\frac{1}{2}\left|C_{s+1}\right|, \quad C_{s} \subset C_{s+1}$, for $s \in\{1, \ldots, k-1\}$.

2. The right fill-up of the cone $\mathcal{C}$ is the collection of dyadic intervals, $\mathcal{R}=$ $\mathcal{R}(I, J)=\bigcup_{s=1}^{k-1} \mathcal{U}_{s+1}$, where $\mathcal{U}_{s+1}=\phi$ if $C_{s}$ is the right half of $C_{s+1}$ and

$$
\mathcal{U}_{s+1}=\left\{J:|J| \geq 2^{-N}, J \subseteq C_{s+1} \backslash C_{s}\right\}
$$

if $C_{s}$ is the left half of $C_{s+1}$.

Proposition 1.1. Let $J_{1}, J_{2} \in T_{N}$ satisfy $J_{1} \preceq J_{2}$, then there exists a (unique) collection $\mathcal{L}=\left\{L_{1}, \ldots, L_{m}\right\}$ of pairwise disjoint dyadic intervals satisfying:

1) $\left|L_{i}\right| \leq \frac{1}{2}\left|L_{i-1}\right|$ if $1 \leq i \leq m-1$;

2) $\left|L_{m-1}\right|=\left|L_{m}\right|($ if $m \geq 2)$;

3a) $\overline{L_{i}}$ and $\overline{L_{i+1}}$ intersect in exactly one point (the left endpoint of $\overline{L_{i+1}}$ );

3b) $L_{i+1}$ lies right of $L_{i}$;

4) $J_{1} \subseteq L_{1}, J_{2}=L_{m}$ such that

$$
\left\{I \in T_{N} ; J_{1} \preceq I \preceq J_{2}\right\}=\mathcal{C}\left(J_{1}, L_{1}\right) \cup \mathcal{R}\left(J_{1}, L_{1}\right) \cup_{i=2}^{m} \mathcal{M}_{i}
$$

where $\mathcal{M}_{i}=\left\{I \in T_{N} ; I \subseteq L_{i}\right\}$.

Proof. Assume first that $J_{1} \subseteq J_{2}$. Then the assertion is that a dyadic interval $I$ satisfies $J_{1} \preceq I \preceq J_{2}$ if and only if $I \in \mathcal{C}\left(J_{1}, J_{2}\right) \cup \mathcal{R}\left(J_{1}, J_{2}\right)$.

The if part is clear. Assume now $J_{1} \preceq I \preceq J_{2}$. Clearly, $I$ cannot be disjoint from $J_{2}$ : otherwise it would have to be to the left of $J_{2}$ and thus to the left of $J_{1}$ contradicting $J_{1} \preceq I$. So $I \subseteq J_{2}$. If $J_{1} \subseteq I$ then by the definition of a cone, $I \in \mathcal{C}\left(J_{1}, J_{2}\right)$. If not, then $I$ lies to the right of $J_{1}$. Then there exists $C_{i_{0}} \in \mathcal{C}\left(J_{1}, J_{2}\right)$, so that $I \subset C_{i_{0}}$ and $I \cap C_{i_{0}-1}=\varnothing$. We then claim that $C_{i_{0}} \backslash C_{i_{0}-1}$ is the right half of $C_{i_{0}}$, and then $I \in \mathcal{R}\left(J_{1}, J_{2}\right)$.

Indeed, if $C_{i_{0}} \backslash C_{i_{0}-1}$ is the left half of $C_{i_{0}}$, then $I$ lies to the left of $C_{i_{0}-1}$ and thus to the left of $J_{1}$, a contradiction. This concludes the proof of this special case and we turn to the general case.

Let $D_{1}$ be the minimal dyadic interval which contains both $J_{1}$ and $J_{2}$. If $D_{1}=J_{2}$ put $m=1$ and $L_{1}=D_{1}$.

If $D_{1}$ strictly contains $J_{2}$ then take the left half of $D_{1}$ and call it $L_{1}$. Observe that $J_{2} \subseteq D_{1} \backslash L_{1}=R_{1}$ where $R_{1}$ is the right half of $D_{1}$.

Let $I$ be the dyadic interval of length $2^{-N}$ which has the same left endpoint as $R_{1}$. Let $D_{2} \subseteq R_{1}$ be the minimal dyadic interval which contains both $I$ and $J_{2}$.

If $D_{2}=J_{2}$ put $m=2, L_{2}=D_{2}$. If $D_{2}$ strictly contains $J_{2}$ then take the left half of $D_{2}$ and call it $L_{2}$.

Observe that $J_{2} \subseteq D_{2} \backslash L_{2}=R_{2}$ where $R_{2}$ is the right half of $D_{2}$.

Continuing in that manner (let $I$ be the dyadic interval of length $2^{-N}$ which has the same left endpoint as $R_{2}$. Let $D_{3} \subseteq R_{2}$ be the minimal dyadic interval which contains both $I$ and $J_{2} \ldots$ ), the process stops after finitely many steps and we get a collection $\mathcal{L}=\left\{L_{1}, \ldots, L_{m}\right\}$ of pairwise disjoint dyadic intervals satisfying 1 
through 4. We are left with the task of proving (1). Clearly, the right-hand side in (1) is contained in the left-hand side. To prove the other containment, note that if $J_{1} \preceq I \preceq J_{2}$ then none of the $L_{i}, i \leq m$ is strictly contained in $I$. Suppose not; then for some $i, D_{i} \subseteq I$ and from $J_{2} \subseteq D_{i} \subseteq I$ we get $J_{2} \prec I$. A contradiction.

It follows that there exists $i_{0}$, so that $I \subseteq L_{i_{0}}$. Suppose not; then using that $\bigcup_{i=1}^{m} L_{i}$ is an interval (not necessarily dyadic) $I$ is either to the right of $L_{m}$ or to the left of $L_{1}$. Hence $I \prec J_{1}$ or $J_{2} \prec I$. A contradiction.

If $i_{0} \geq 2$ then $I \in \mathcal{M}_{i_{0}}$ and we are done, so, we may suppose $J_{1} \preceq I \preceq L_{1}$. This is exactly the special situation described in the beginning of this proof and we conclude that in this case $I \in \mathcal{C}\left(J_{1}, L_{1}\right) \cup \mathcal{R}\left(J_{1}, L_{1}\right)$.

Next we would like to describe the interaction between two successive order intervals. Let $I_{1} \prec I_{2} \prec J_{1} \prec J_{2}$ and put

$$
\mathcal{B}_{1}=\left\{I \in T_{N} ; I_{1} \preceq I \preceq I_{2}\right\}, \quad \mathcal{B}_{2}=\left\{I \in T_{N} ; J_{1} \preceq I \preceq J_{2}\right\} .
$$

Let $\mathcal{L}_{1}=\left\{L_{1}^{1}, \ldots, L_{m(1)}^{1}\right\}$ be the maximal (with respect to inclusion) elements of $\mathcal{B}_{1}$ as given in Proposition 1.1 and let $\mathcal{L}_{2}=\left\{L_{1}^{2}, \ldots, L_{m(2)}^{2}\right\}$ be the maximal elements of $\mathcal{B}_{2}$. Let $B_{i}=\bigcup_{I \in \mathcal{B}_{i}}$. Clearly $B_{1}$ and $B_{2}$ may intersect.

Lemma 1.2. a) $B_{1}$ may have intersection only with $L_{1}^{2}$; i.e.,

$$
\left(B_{2} \backslash L_{1}^{2}\right) \cap B_{1}=\varnothing .
$$

b) If $B_{1} \cap B_{2} \neq \varnothing$ then $L_{1}^{2} \supseteq L_{m(1)}^{1}$.

Proof. Note that if $I \preceq J_{1}$ then $I \cap L_{j}^{2}=\phi$ for $j \geq 2$. Indeed, if $I \cap L_{j}^{2} \neq 0$ for some $j \geq 2$ then either

Case 1 . $I \subseteq L_{j}^{2}$ and then $I$ lies to the right of $L_{1}^{2}$ and $L_{1}^{2}$ lies above $J_{1}$. Hence by the definition of the order $J_{1} \preceq I$, a contradiction. Or,

Case 2. $I \supset L_{j}^{2}$ and then $J_{1} \prec L_{j}^{2} \prec I$. A contradiction again.

This proves a.

To prove b, note that $L_{m(1)}^{1}=I_{2} \prec J_{1} \preceq L_{1}^{2}$. If $L_{m(1)}^{1} \nsubseteq L_{1}^{2}$ then necessarily $L_{m(1)}^{1}$ is to the left of $L_{1}^{2}$. But then $L_{j}^{1}$ is to the left of $L_{1}^{2}$ for all $j$ and $B_{1} \cap L_{1}^{2}=\varnothing$. Since also $B_{1} \cap\left(B_{2} \backslash L_{1}^{2}\right)=\varnothing$ we get a contradiction to $B_{1} \cap B_{2} \neq \varnothing$.

It follows from the lemma that if an interval $I$ from $\mathcal{B}_{2}$ intersects $B_{1}$ then it belongs to either $\mathcal{C}\left(J_{1}, L_{1}^{2}\right)$ or to $\mathcal{R}\left(J_{1}, L_{1}^{2}\right)$. The second possibility is easily ruled out since in this case $I$ lies to the right of $J_{1}$ while every interval in $\mathcal{B}_{1}$ lies to the left or below $J_{1}$, and one gets (using part b of the lemma for the 'moreover' part)

Proposition 1.3. If $B_{1} \cap B_{2} \neq \varnothing$ then every interval in $\mathcal{B}_{2}$ which intersects $B_{1}$ belongs to $\mathcal{C}\left(J_{1}, L_{1}^{2}\right)$. Moreover,

$$
\left\{I \in \mathcal{B}_{2} ; I \cap B_{1} \neq \varnothing\right\}=\mathcal{C}\left(J_{1}, L_{1}^{2}\right) \cap \mathcal{C}\left(I_{2}, L_{1}^{2}\right) .
$$

2. BLOCK BASES OF $\left\{h_{I}\right\}_{I \in T_{N}}$ WITH RESPECT TO THE ORDER $\preceq$

Given $M \in \mathbf{N}$ and elements of $T_{N}$,

$$
I_{1} \prec J_{1} \prec I_{2} \prec J_{2} \prec \cdots \prec I_{M} \prec J_{M},
$$

consider the order intervals $\mathcal{B}_{i}=\left\{I \in T_{N} ; I_{i} \preceq I \preceq J_{i}\right\}$. Let $\mathcal{L}_{i}=\left\{L_{1}^{i}, \ldots, L_{m(i)}^{i}\right\}$ be the maximal (with respect to inclusion) elements of $\mathcal{B}_{i}$ as given in Proposition 
1.1. Put also $\mathcal{C}_{i}=\mathcal{C}\left(I_{i}, L_{1}^{i}\right)$ - the cone of intervals between $I_{i}$ and $L_{1}^{i}$, and let $\mathcal{G}_{i}=\mathcal{B}_{i} \backslash \mathcal{C}_{i}$. Note that, by Proposition 1.3,

a) The sets $\bigcup_{I \in \mathcal{G}_{i}} I, i=1,2, \ldots, M$ are pairwise disjoint, and

b) the sets $L_{1}^{i}=\bigcup_{I \in \mathcal{C}_{i}} I, i=1,2, \ldots, M$ are nested; i.e., for $i<j$, either $L_{1}^{i} \cap L_{1}^{j}=\varnothing$ or $L_{1}^{i} \subset L_{1}^{j}$. In the latter case $\left|L_{1}^{i}\right| \leq\left|L_{1}^{j}\right| / 2$.

Now let $\left\{Z_{i}\right\}_{i=1}^{M}$ be a block basis of $\left\{h_{I}\right\}$ with respect to the blocking $\left\{\mathcal{B}_{i}\right\}$; i.e.,

$$
Z_{i}=\sum_{I \in \mathcal{B}_{i}} \alpha_{I} h_{I}, \quad i \leq M
$$

Consider

$$
X_{i}=\sum_{I \in \mathcal{C}_{i}} \alpha_{I} h_{I}, \quad Y_{i}=\sum_{I \in \mathcal{G}_{i}} \alpha_{I} h_{I}
$$

Then $X_{i}+Y_{i}=Z_{i}$. Clearly, the $Y_{i}^{\prime}$ 's have disjoint supports (with respect to the underlying measure space - the interval $[0,1]$ ) and thus are equivalent to multiples of the canonical basis elements of $\ell_{p}^{M}$. To analyze the $\left\{X_{i}\right\}$ we must now study more carefully the cones $\left\{\mathcal{C}_{i}\right\}$ and their interaction. We start with the following observation which is self evident.

Observation. Let $\mathcal{C}_{1}=\left\{I_{1}, \ldots, I_{k}\right\}$ with $I_{1} \subset I_{2} \subset \ldots I_{k},\left|I_{i}\right|=\frac{1}{2}\left|I_{i+1}\right|$, and $\mathcal{C}_{2}=\left\{J_{1}, \ldots, J_{l}\right\}$ with $J_{1} \subset J_{2} \subset \ldots J_{l},\left|J_{i}\right|=\frac{1}{2}\left|J_{i+1}\right|$ be two cones of intervals consisting of distinct intervals (i.e., $I_{i} \neq J_{j}$ for all $i, j$ ). Suppose that $J_{l} \subset I_{k}$ and put, for convenience of notation, $I_{0}=$ the right half of $I_{1}$ and $I_{-1}=\varnothing$; then there exists $0 \leq i \leq k$ such that $J_{l} \subseteq I_{i} \backslash I_{i-1}$.

Let $\mathcal{C}=\left\{I_{1}, \ldots, I_{k}\right\}$ be a cone of intervals and put, for $i=1, \ldots, k, D_{i}=$ $I_{i} \backslash I_{i-1}$. Then the $D_{i}, i=1, \ldots, k$, are disjoint dyadic intervals such that $\left|D_{i}\right|=$ $\frac{1}{2}\left|I_{i}\right|$ and $D_{i} \subset I_{i}$. Also, $\left|I_{k}\right| \leq 2\left|\bigcup_{i=1}^{k} D_{i}\right| \leq\left|I_{k}\right|$.

Returning to the $X_{i}$ 's, denoting $\mathcal{C}_{i}=\left\{I_{1}^{i}, \ldots, I_{k(i)}^{i}\right\}$ and by $\mathcal{D}_{i}=\left\{D_{1}^{i}, \ldots, D_{k(i)}^{i}\right\}$ the collection of intervals obtained from $\mathcal{C}_{i}$ by the process above, put

$$
\phi_{i}=\sum_{j=1}^{k(i)} \alpha_{I_{j}^{i}} h_{D_{j}^{i}} .
$$

(To obtain $\phi_{i}$ from $X_{i}$ we just replace $h_{I_{j}^{i}}$ by $h_{D_{j}^{i}}$.)

Lemma 2.1. The map $X_{i} \mapsto \phi_{i}, i=1, \ldots, M$, extends to an isomorphism from $\left(\operatorname{span}\left\{X_{i}\right\},\|\cdot\|_{L_{p}}\right)$ to $\left(\operatorname{span}\left\{\phi_{i}\right\},\|\cdot\|_{L_{p}}\right)$ where the isomorphism constant depends on $p$ alone, $1<p<\infty$.

Proof. The assertion follows easily from a theorem of Semenov [S]. We prefer to give an ad hoc proof.

Extend the map $X_{i} \mapsto \phi_{i}, i=1, \ldots, M$, to the map given by $T_{p}: h_{I_{j}^{i}} \mapsto h_{D_{j}^{i}}$ from $\left(\operatorname{span}\left\{h_{I_{j}^{i}}^{k}\right\}_{j=1, i=1}^{k(i), M},\|\cdot\|_{L_{p}}\right)$ to $\left(\operatorname{span}\left\{h_{D_{j}^{i}}\right\}_{j=1, i=1}^{k(i), M},\|\cdot\|_{L_{p}}\right)$. Note that the square function $\left(\sum\left(a_{j}^{i}\right)^{2}\left(h_{I_{j}^{i}}\right)^{2}\right)^{1 / 2}$ pointwise dominates $\left(\sum\left(a_{j}^{i}\right)^{2}\left(h_{D_{j}^{i}}\right)^{2}\right)^{1 / 2}$ and consequently $T_{p}$ is bounded in $L_{p}, 1<p<\infty$, with bound depending on $p$ alone.

The unconditionality of the Haar system also implies that the dual space to $\left(\operatorname{span}\left\{h_{I_{j}^{i}}\right\}_{j=1, i=1}^{k(i), M},\|\cdot\|_{L_{p}}\right)$ can be naturally identified with (span $\left\{h_{I_{j}^{i}}\right\}_{j=1, i=1}^{k(i), M}$, $\left.\|\cdot\|_{L_{q}}\right), \frac{1}{p}+\frac{1}{q}=1$, with equivalent norms, and a similar identification can be done 
between the dual of $\left(\operatorname{span}\left\{h_{D_{j}^{i}}\right\}_{j=1, i=1}^{k(i), M},\|\cdot\|_{L_{p}}\right)$ and $\left(\operatorname{span}\left\{h_{D_{j}^{i}}\right\}_{j=1, i=1}^{k(i), M},\|\cdot\|_{L_{q}}\right)$. Since the conjugate operator to $T_{p}$ is $T_{q}^{-1}$, we are done.

We shall show in Proposition 2.3 below that, for $2<p<\infty,\left\{X_{i}\right\}$ is well equivalent to an appropriately normalized subsequence of a permutation of the Haar system and thus we'll get the following theorem.

Theorem 2.2. For every $2<p<\infty$ there exists a constant $K_{p}, 1 \leq K_{p}<\infty$, such that, for all $N$, every block basis of $\left\{h_{I}\right\}_{I \in T_{N}}$ (with respect to the order $\preceq$ ) is equivalent, with constant at most $K_{p}$, to a sequence of the kind $\left\{a_{i} x_{i} \oplus b_{i} e_{i}\right\}_{i=1}^{n}$, where $\left\{x_{i}\right\}_{i=1}^{n}$ is a subsequence of a permutation of $\left\{h_{I}\right\}_{I \in T_{N}}$ and $\left\{e_{i}\right\}_{i=1}^{n}$ is the unit vector basis in $\ell_{p}^{n}$.

It may be instructive to recall here the local version of the Gamlen-Gaudet Theorem proved by the first named author in $[\mathrm{M}]$.

Theorem. For every $1<p<\infty$ there exists a constant $K_{p}, 1 \leq K_{p}<\infty$, such that, for all $N$, every subsequence of $\left\{h_{I}\right\}_{I \in T_{N}}$ (of length $k$, say) spans a space isomorphic, with constant at most $K_{p}$, to $\ell_{p}^{k}$.

Problem. Can Theorem 2.2 (and Theorem 3.1 below) be strengthened to show that for every $1<p<\infty$ there exists a constant $K_{p}, 1 \leq K_{p}<\infty$, such that, for all $N$, every block basis of $\left\{h_{I}\right\}_{I \in T_{N}}$ (with respect to the order $\preceq$ ) of length $k$, say, spans a space isomorphic, with constant at most $K_{p}$, to $\ell_{p}^{k}$ ?

An easy consequence of Theorem 2.2 (see the proof of Theorem 3.1) is that there exists a subset $A \subseteq\{1, \ldots, M\}$ with $|A| \geq M / 2$ and such that $\left\{Z_{i}\right\}_{i \in A}$ is well equivalent to the unit vector basis of $\ell_{p}^{|A|}, 2<p<\infty$. This turns out to be true also for $1<p<2$ and is easier to deduce than Theorem 2.2. We shall do it in the next section. Of course this is enough for the solution of problem 3.A in [DS]. We continue now with the preparations to the proof of Theorem 2.2.

Let $\mathcal{E}$ be the collection of the largest intervals in the cones $\mathcal{C}_{i}, i=1, \ldots, M$, i.e., $\mathcal{E}=\left\{I_{k(i)}^{i}\right\}$. Let $G_{1}(\mathcal{E})$ be the subcollection of maximal (with respect to inclusion) intervals out of $\mathcal{E}$. Note that any two distinct intervals in $G_{1}(\mathcal{E})$ are disjoint. Define, by induction, $G_{k}(\mathcal{E})$ to be the subcollection of maximal intervals out of $\mathcal{E} \backslash \bigcup_{i=1}^{k-1} G_{i}(\mathcal{E})$.

Given $C=I_{k(i)}^{i} \in \mathcal{E}$ denote $\mathcal{D}(C)=\mathcal{D}_{i}$ (that is the collection of intervals obtained as successive differences of the intervals of the cone that $C$ is the maximal element of). Denote also by $\mathcal{D}^{1 / 2}(C)$ the collection of the left and right halves of the intervals in $\mathcal{D}(C)$ and put

$$
\mathcal{H}_{k}=\bigcup_{C \in G_{k}(\mathcal{E})} \mathcal{D}(C), \quad \mathcal{H}_{k}^{1 / 2}=\bigcup_{C \in G_{k}(\mathcal{E})} \mathcal{D}^{1 / 2}(C), \quad k=1,2, \ldots
$$

We now define an increasing sequence of $\sigma$-fields by

$$
\mathcal{E}_{1}=\mathcal{A}\left\{\mathcal{H}_{1}\right\}, \quad \mathcal{E}_{k}=\mathcal{A}\left\{\mathcal{E}_{k-1}, \mathcal{H}_{k}, \mathcal{H}_{k-1}^{1 / 2}\right\}
$$

where $\mathcal{A}\{\mathcal{H}\}$ denotes the $\sigma$-field generated by $\mathcal{H}$. We are now ready to prove

Proposition 2.3. Let $\mathcal{C}_{i}=\left\{I_{1}^{i}, \ldots, I_{k(i)}^{i}\right\}, i=1, \ldots, M$, be pairwise disjoint cones of intervals. Using the notation above (for the $\mathcal{D}$ 's), let $\phi_{i}=\sum_{j=1}^{k(i)} \alpha_{I_{j}^{i}} h_{D_{j}^{i}}$, $i=1, \ldots, M$, be a normalized, in $L_{p}$, sequence. Then $\left\{\phi_{i}\right\}_{i=1}^{M}$ is equivalent, with 
constant depending only on $p, 2<p<\infty$, to a permutation of a subsequence of the normalized Haar system.

Proof. Reindex the sequence $\left\{\phi_{i}\right\}$, using $\mathcal{E}$ as an index set, by putting $\phi_{i}=\phi_{C}$ if $C=I_{k(i)}^{i}$. Then for $C \in G_{k}(\mathcal{E})$ we have by the Observation above

1) $\phi_{C}$ is $\mathcal{E}_{k+1}$ measurable,

2) $\mathbf{E}\left(\phi_{C} \mid \mathcal{E}_{k}\right)=0$

3) $C \neq C^{\prime} \in G_{k}(\mathcal{E})$ implies supp $\phi_{C} \cap \operatorname{supp} \phi_{C^{\prime}}=\varnothing$.

By Burkholder's theorem (see [B, (21.5)]), we have for all scalars $\left\{a_{C}, C \in \mathcal{E}\right\}$

$$
\begin{aligned}
& \left\|\sum_{k} \sum_{C \in G_{k}(\mathcal{E})} a_{C} \phi_{C}\right\|_{L_{p}} \stackrel{C_{p}}{\sim} \\
& \stackrel{C_{p}}{\sim} \max \left\{\left\|\left(\sum_{k} \sum_{C \in G_{k}(\mathcal{E})} a_{C}^{2} \mathbf{E}\left(\phi_{C}^{2} \mid \mathcal{E}_{k}\right)\right)^{1 / 2}\right\|_{L^{p}},\left(\sum_{C \in \mathcal{E}}\left|a_{C}\right|^{p}\right)^{1 / 2}\right\} .
\end{aligned}
$$

To prove the proposition it is thus enough to prove that, for every $k$ and every $C \in G_{k}(\mathcal{E})$, there exists an $I \in \mathcal{D}(C)$ such that ratio of the two functions $\mathbf{E}\left(\phi_{C}^{2} \mid \mathcal{E}_{k}\right)$ and $\mathbf{E}\left(h_{I}^{2} /\left\|h_{I}\right\|_{p}^{2} \mid \mathcal{E}_{k}\right)$ is bounded and bounded away from 0 by constants depending only on $p$. This follows easily, by rearranging and rescaling, from the following lemma.

Lemma 2.4. Let $p>2$, let $D_{m}=\left[2^{-m}, 2^{-(m-1)}\right)$ and let $\beta_{m}>0$ so that $\sum_{m=1}^{n}\left|\beta_{m}\right|^{p}=1$. Let $\bar{h}_{n}$ be the $L_{p}$-normalized Haar function having $D_{m}$ as support. Then for $\phi=\sum_{m=1}^{n} \beta_{m} \bar{h}_{m}$ we have

$$
2^{-n\left(1-\frac{2}{p}\right)} \leq \int_{0}^{1}|\phi|^{2} d t \leq 1 .
$$

Consequently, there exists an $1 \leq m \leq n$ satisfying

$$
1 \leq \int_{0}^{1}|\phi|^{2} d t / \int_{0}^{1}\left|\bar{h}_{m}\right|^{2} d t \leq 2^{\left(1-\frac{2}{p}\right)} .
$$

Proof of Lemma 2.4. The right-hand side inequality is clear. To prove the left-hand side, notice that this is the same problem as finding the (square of the) minimal $L_{2}$ norm of a function which is constant on each of the $D_{m}$ 's and has $L_{p}$ norm equal to 1 . The minimum is attained for the function with minimal support, i.e., for the characteristic function of $D_{n}$ normalized in $L_{p}$ and thus is equal to $2^{-n\left(1-\frac{2}{p}\right)}$.

Let $1 \leq m \leq n$ be such that

$$
2^{-m\left(1-\frac{2}{p}\right)} \leq \int_{0}^{1}|\phi|^{2} d t \leq 2^{-(m-1)\left(1-\frac{2}{p}\right)}
$$

then

$$
\begin{aligned}
1 \leq \int_{0}^{1}|\phi|^{2} d t / 2^{-m\left(1-\frac{2}{p}\right)} & =\int_{0}^{1}|\phi|^{2} d t / \int_{0}^{1}\left|\bar{h}_{m}\right|^{2} d t \\
& \leq 2^{\left(1-\frac{2}{p}\right)} \int_{0}^{1}|\phi|^{2} d t / 2^{-(m-1)\left(1-\frac{2}{p}\right)} \leq 2^{\left(1-\frac{2}{p}\right)} .
\end{aligned}
$$

This ends the proof of the lemma and thus of Proposition 2.3 and Theorem 2.2. 


\section{THE CASE $1<p<2$}

Here we prove:

Theorem 3.1. For every $1<p<2$ there exists a constant $K_{p}, 1 \leq K_{p}<\infty$, such that, for all $N$, every normalized block basis $\left\{Z_{i}\right\}_{i=1}^{M}$ of $\left\{h_{I}\right\}_{I \in T_{N}}$ (with respect to the order $\preceq)$ contains a subsequence $\left\{Z_{i}\right\}_{i \in A}$ with $|A| \geq M / 2$ which is equivalent, with constant at most $K_{p}$, to the unit vector basis of $\ell_{p}^{|A|}$.

An identical statement holds for $2<p<\infty$ and its proof follows that of the proof below using Theorem 2.2. With a bit more effort one can also avoid referring to Theorem 2.2. We do not reproduce this case here.

For the proof of Theorem 3.1 we shall need only the first two paragraphs in Section 2, that is up to, and not including, the Observation. We start with a lemma similar to one used in $([\mathrm{F}, \mathrm{p} .156])$.

Lemma 3.2. Let $T$ be a finite tree with partial order $<$. Put

$$
A_{0}=\{t \in T ; t \text { is a minimal element }\}
$$

and

$$
A_{1}=\left\{t \in T ; t \notin A_{0} \text { and there is exactly one maximal } s \text { with } s<t\right\} .
$$

Then $|T|<2\left|A_{0}\right|+\left|A_{1}\right|$.

Proof. Assume first $A_{1} \neq \emptyset$. Let $t \in A_{1}$ and let $s$ be the unique maximal element with $s<t$. Put $T^{\prime}=T \backslash\{s\}$ and let $A_{0}^{\prime}$ and $A_{1}^{\prime}$ be the corresponding " $A_{0}$ " and " $A_{1}$ " associated to this new tree. Note that $\left|A_{0}^{\prime}\right|=\left|A_{0}\right|$. (If $s \in A_{0}$ then $t \in A_{0}^{\prime}$ while if $s \notin A_{0}, A_{0}^{\prime}=A_{0}$.) Note also that $\left|A_{1}^{\prime}\right|=\left|A_{1}\right|-1$. (If $s \notin A_{1}$ then $A_{1}^{\prime}=A_{1} \backslash\{t\}$ while if $s \in A_{1}$ then $A_{1}^{\prime}=A_{1} \backslash\{s\}$.) We thus reduce the problem to proving $\left|T^{\prime}\right|<2\left|A_{0}^{\prime}\right|+\left|A_{1}^{\prime}\right|$.

Continuing reducing the tree in this fashion we finally reach a tree for which $A_{1}=\emptyset$, i.e., it branches everywhere : every node has at least two offsprings. For such a tree the conclusion is well known and easy.

Proof of Theorem 3.1. We follow the notation of the beginning of Section 2. Since the sequence $\left\{Y_{i} /\left\|Y_{i}\right\|\right\}_{i=1}^{M}$ is isometrically equivalent to the unit vector basis of $\ell_{p}^{M}$, it is enough to prove that there exists a subset $A \subseteq\{1, \ldots, M\}$ with $|A| \geq M / 2$ for which $\left\{X_{i} /\left\|X_{i}\right\|\right\}_{i \in A}$ is equivalent, with constant depending only on $p$, to the unit vector basis of $\ell_{p}^{|A|}$.

Recall that the supports (with respect to the underlying measure space) of the $X_{i}$ 's are the sets $L_{1}^{i}, i=1, \ldots, M$, and they are nested; i.e., any two of them are either disjoint or one is contained in the other. Let

$$
A_{0}=\left\{i ; L_{1}^{i} \text { contains no other } L_{1}^{j}\right\}
$$

and

$$
A_{1}=\left\{i ; L_{1}^{i} \text { contains exactly one other maximal } L_{1}^{j}\right\}
$$

(i.e., $\exists j \neq i$ with $L_{j} \subset L_{i}$ but no two disjoint $L_{j}$ 's with this property). By Lemma $3.2\left|A_{0}\right|+\left|A_{1}\right| \geq M / 2$. The set $A=A_{0} \cup A_{1}$ can be decomposed into sets $B_{k}$, $k=1, \ldots, K$, with $L_{1}^{i} \cap L_{1}^{j}=\phi$ if $i$ and $j$ are in different $B_{k}$ 's and $L_{1}^{i} \subset L_{1}^{j}$ or $L_{1}^{j} \subset L_{1}^{i}$ if they are in the same one. It is thus enough to prove that $\left\{X_{i} /\left\|X_{i}\right\|\right\}_{i \in B_{k}}$ is equivalent, with constant depending only on $p$, to the unit vector basis of $\ell_{p}^{\left|B_{k}\right|}$ and 
thus, without loss of generality that, if $L_{1}^{1} \subset L_{1}^{2} \subset \cdots \subset L_{1}^{m}$, then $\left\{X_{i} /\left\|X_{i}\right\|\right\}_{i=1}^{m}$ is well equivalent to the unit vector basis of $\ell_{p}^{m}$.

Let $1<i \leq m$ and let $\mathcal{C}_{i}=\left\{I_{1} \ldots, I_{r}\right\}$ be the cone of intervals associated with $X_{i}$. Note that there exists a $1 \leq s \leq r$ with $L_{i-1} \subseteq I_{s} \backslash I_{s-1}\left(I_{0}\right.$ is one of the two halves of $\left.I_{1}\right)$. Clearly $\left\|X_{i \mid[0,1] \backslash L_{i-1}}\right\| \geq c\left\|X_{i}\right\|$ for some constant $c>0$ depending only on $p$. Now a simple square function argument shows that the sequence $\left\{X_{i}\right\}$ well

dominates the sequence $\left\{X_{i \mid[0,1] \backslash L_{i-1}}\right\}$. This finishes the proof since the functions $X_{i \mid[0,1] \backslash L_{i-1}}, i=1, \ldots, m$, are disjoint.

\section{REFERENCES}

[B] D.L. Burkholder, Distribution function inequalities for martingales, Ann. Prob. 1 (1973), 19-43. MR 51:1944

[DS] L.E. Dor and T. Starbird, Projections of $L_{p}$ onto subspaces spanned by independent random variables, Compositio Math. 39 (1979), 141-175. MR 82e:46043

[F] C.L. Fefferman, The uncertainty principle, Bull. AMS 9 (1983), 129-206. MR 85f:35001

[JMST] W.B. Johnson, B. Maurey, G. Schechtman, and L. Tzafriri, Symmetric structures in Banach spaces, Mem. AMS 217 (1979). MR 82j:46025

[M] P.F.X. Müller, A local version of a result of Gamlen-Gaudet, Israel J. Math. 63 (1988), 212-222. MR 89m:46016

[S] E.M. Semenov, On the equivalence in $L_{p}$ of rearrangements of the Haar system, English translation in Soviet Math. Dokl. 19 (1978), 1292-1294.

Institut für Mathematik, J. Kepler Universität, 4040 Linz, Austria

E-mail address: paul.mueller@jk.uni-linz.ac.at

Current address: Department of Mathematics, Yale University, 10 Hill House Avenue, New Haven, Connecticut 06520

E-mail address: muller@math.yale.edu

Department of Theoretical Mathematics, The Weizmann Institute of Science, ReHOVOT 76100, ISRAEL

E-mail address: mtschech@weizmann.weizmann.ac.il 